\title{
PADRÃO DE COMUNICAÇÃO ENTRE PACIENTES NUM HOSPITAL GOVERNAMENTAL BRASILEIRO*
}

\author{
Isabel Amélia Costa Mendes ** \\ Maria Auxiliadora Trevizan ** \\ Namie Okino Sawada *** \\ Cristina Maria Galvão *** \\ Maria Suely Nogueira ***
}

MENDES, I.A.C. et al. Padrão de comunicação entre pacientes num hospital governamental brasileiro. Rev. Esc. Enf. USP., v. 27, n.3, p. 403-12, dez. 1993.

Numa situação de interação verbal em enfermagem hospitalar, as autoras exploram o contexto do paciente com o objetivo de verificar o padrão de comunicação dos pacientes entre si. Os dados foram coletados através de observaçāo direta do comportamento verbal de uma amostra de 10 pacientes com seus companheiros de enfermaria. Do seu registro em formulário, os dados foram analisados por juizes $e$ classificados à luz do instrumento de Bales. Os resultados apontam para o seguinte padrão de comunicaçāo: $83,1 \%$ das unidades de interação foram classificadas na área neutra, 8,8\% na área 8ócio-emocional positiva e 8,1\% na área sócio-emocional negativa. As autoras discutem tais resultados, estimulando os enfermeiros a repensarem o valor da comunicação no processo de enfermagem.

UNITERMOS: Comunicaçāo. Enfermagem. Relaçōes enfermeira-paciente.

A centralidade da comunicação na prestação da assistência de enfermagem vem sendo reconhecida, de forma crescente, desde o advento da enfermagem moderna. A relevância da comunicação efetiva no relacionamento enfermeira-paciente registrada na descrição de NIGHTINGALE ${ }^{17}$, já demonstrava a preocupação e a dificuldade de se estabelecer uma comunicação real com o paciente.

O enfoque da comunicação entre os elementos da equipe de enfermagem e pacientes tem sido objeto de estudos desde a década de cinqüen-

\footnotetext{
- Trabalho comunicado na $3^{*}$ Conferência de Investigação em Enfermagem, Lisboa, Portugal, novembro de 1991 .

- Enfermeira, Doutora e Livre-Docente em Enfermagem. Professor Titular da Escola de Enfermagem de Ribeirão Preto - Universidade de Sảo Paulo, Brasil.

*.* Enfermeira e Mestre , Enfermagem. Professor-Assistente da Escola de Enfermagem de Ribeiráo Preto - Universidade de Sảo Paulo, Brasil.
} 
ta, surgindo como uma reação a grande ênfase dada ao aspectos técnicos da profissão ${ }^{14}$.

A enfermagem tem se caracterizado teoricamente como uma profissão da saúde que prioriza a comunicação com o paciente, como essência do processo da assistência que presta. Entretanto, estudos comprovam que na prática essa correspondência nāo se confirma.

Verifica-se, nos estudos que tratam da comunicação em enfermagem, que os enfermeiros tem se utilizado de uma variedade de sistemas de análise na busca do perfil de interação enfermeiro-paciente em diferentes situações de relacionamento verbal e/ou não ver$\mathrm{bal}^{3,4,5,6,8,10,13,18,19,21}$, bem como do perfil de interação do paciente com o pessoal de enfermagem ${ }^{7,11,12}$ ou das interaçōes do paciente psiquiátri$\mathrm{co}^{15}$ com as enfermeiras e alunos de enfermagem ${ }^{20}$.

Independentemente do instrumento utilizado, depreende-se destes estudos que os enfermeiros se interessam e se preocupam com esta área de pesquisa procurando obter algum resultado e delinear o foco de intervenção para melhorar o nível e a qualidade de comunicação na enfermagem. Isto pode ser comprovado pela exaltação das falhas no conteúdo e no processo de comunicação com os pacientes, presente nestes estudos.

Em consonância com este panorama, $\mathrm{MENDES}^{9}$ em pesquisa anterior, utilizando-se da estrutura teórica do paradigma categorial de Bales e o método de análise do processo de interação em situações de enfermagem, chamou a atenção que, ao lado da falta de ressonância dos preceitos teóricos encontrados na prática da comunicação entre equipe de enfermagem e paciente, os pacientes numa situação de internação hospitalar buscam ajuda uns com outros, ocorrendo portanto a comunicação com seus pares.

Assim, propomo-nos neste estudo, analisar o perfil e o nível de interação entre pacientes em uma situação de internação hospitalar.

\section{METODOLOGLA}

A amostra investigada no presente estudo é proveniente de um banco de dados constituído em 1986 por MENDES $^{9}$, que envolve a interação verbal e não-verbal de ocupacionais e profíssionais da equipe de saúde de um hospital governamental de grande porte do município de Ribeirão Preto.

Para este estudo foram extraídos para análise apenas os dados resultantes da comunicação verbal de dez pacientes com seus pares durante cinco dias consecutivos, a contar do momento da internação.

A técnica utilizada foi a observação direta e registro do comportamento verbal dos pacientes selecionados com seus pares. Cada um dos dez pacientes foi submetido a observação por um período aproximado de quarenta horas, englobando os turnos manhā e tarde, sendo os dados 
registrados em formulários por seis observadores especialmente treinados para esta finalidade.

As interações verbais documentadas foram analisadas e classificadas por três juízes à luz do Instrumento de Bales: "Interaction Process Analysis"2.

Para que esta classificação fosse possível, os juízes foram previamente treinados, através de um programa cuja descrição encontra-se detalhada no trabalho de MENDES ${ }^{9}$.

O Instrumento de Bales (Ver Figura 1 na página seguinte) é composto por doze categorias, as quais guiaram a classificação das unidades de interação efetuada pelos juízes. As três primeiras categorias referemse às reaçōes positivas; as seis intermediárias são relativas a tentativas de resposta (4,5 e 6) e a questōes ( 7,8 e 9); as três últimas abrangem reaçōes negativas.

\section{RESULTADOS E DISCUSSÃO}

A matriz de interação entre pacientes, tendo como foco os dez sujeitos alvo deste estudo, encontra-se registrada na Tabela 1 que ilustral a freqüência das unidades de interação emitidas $(E)$ e recebidas $(R)$, segundo as categorias de Bales, indicando que as unidades de interação estão concentradas na Categoria 6 - Dá informação, seguidas pelas Categorias 5 - Dá opiniāo e 7 - Pede informação. Dentre as restantes, a de maior freqüência é a Categoria 11 - Mostra tensão, emitidas pelos pacientes estudados numa freqüência de 130 unidades.

A concentração de interaçōes observadas, nas categorias 6, 5, e 7, mostra o direcionamento dos pedidos e fornecimento de informaçóes do paciente ao seu próprio par e não para o grupo de enfermagem. Estes resultados indicam a necessidade de informação sentida pelo paciente $e$ nos leva a acreditar que possivelmente os componentes do grupo de enfermagem estão preocupados em perguntar e informar apenas o que lhes interessa para o cumprimento de suas funçōes técnicas. No entanto, as informaçōes que os pacientes desejam estão sendo fornecidas por seus companheiros.

Este dado reforça mais uma vez os resultados de pesquisas anteriores que evidenciaram que a interação entre enfermeiros ${ }^{10,13}$, auxiliares de enfermagem ${ }^{11} \mathrm{e}$ atendentes de enfermagem ${ }^{12}$ com os pacientes, têm em vista a solução de problemas desses profissionais e ocupacionais, ou seja, - cumprimento de suas tarefas calçado numa relação mecanizada e despersonalizada. Isto pode estar sugerindo que a esfera de interesses do grupo da enfermagem pode não estar coincidindo com as necessidades sentidas pelos pacientes, que para serem atendidas dependem de comunicação com os elementos desse grupo. 
Figura 1 - Sistema de Categorias Observacionais de Bales

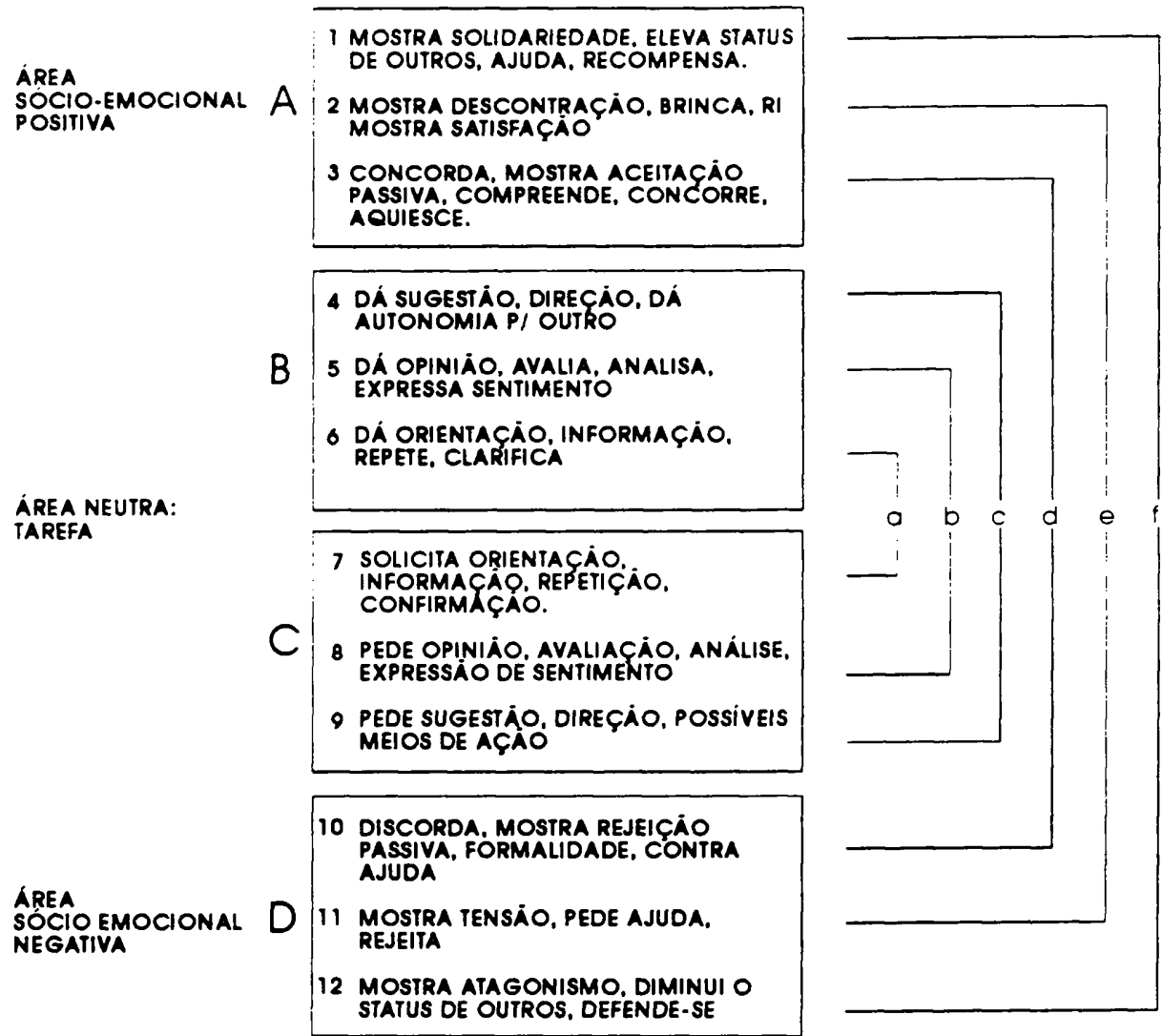

\section{CHAVE}
A - REACÕEs POSITIVAS
B - TENTÁTIVA DE RESPOSTA
C - QUETÓES
D - REAÇÓES

a - Problemas de Comunicaçāo

b - Problemas de Avaliaçáo

c- Problemas de Controle

d - Problemas de Decisáo

- Problemas de Redução de Tensão

f- Problemas de Reintegração

BALES, ROBERT F. INTERACTION PROCESS ANALYSIS, CAMBRIDGE, MASSACHUSETTS, ADDISON-WESLEY PRESS, INC. 1950, p.9. 
TABELA 1 - Distribuição das Unidades de Interação Emitidas e Recebidas pelos pacientes estudados e por outros pacientes, segundo as categorias de Bales.

\begin{tabular}{|c|c|c|c|c|c|c|c|c|c|c|c|c|}
\hline \multirow{2}{*}{$\begin{array}{l}\text { CATEGORLA } \\
\text { DE BALES }\end{array}$} & \multirow{2}{*}{$\begin{array}{l}\text { DIREÇĀO DA } \\
\text { INTERAÇȦO }\end{array}$} & \multicolumn{10}{|c|}{ PACIENTES } & \multirow[t]{2}{*}{ TOTAL } \\
\hline & & 1 & 2 & 3 & 4 & 6 & 6 & 7 & 8 & 8 & 10 & \\
\hline \multirow[t]{2}{*}{1} & $\mathbf{E}$ & 23 & 4 & 5 & 1 & 6 & 1 & 23 & . & 11 & 11 & 85 \\
\hline & $\mathbf{R}$ & 7 & 7 & 9 & 1 & 2 & 3 & 6 & 4 & 7 & 7 & 53 \\
\hline \multirow[t]{2}{*}{2} & $\mathbf{E}$ & 6 & 1 & 7 & - & 16 & - & 14 & 1 & . & & 50 \\
\hline & $\mathbf{R}$ & 1 & - & 2 & . & 16 & - & 13 & - & . & 4 & 36 \\
\hline \multirow[t]{2}{*}{3} & $\mathbf{E}$ & 13 & 5 & 11 & - & 5 & 7 & 4 & 1 & 6 & 12 & 64 \\
\hline & $\mathbf{R}$ & 5 & 3 & 10 & . & 6 & 4 & 16 & - & 3 & 7 & 54 \\
\hline \multirow{2}{*}{4} & $\mathbf{E}$ & 5 & 1 & 9 & - & 1 & 2 & 3 & - & 2 & & \\
\hline & $\mathbf{R}$ & 7 & 4 & 1 & 9 & 3 & 4 & 2 & - & $\frac{2}{-}$ & 19 & 49 \\
\hline & & & & & & & & & & & & \\
\hline \multirow[t]{2}{*}{5} & $\mathbf{E}$ & 21 & 48 & 29 & 3 & 5 & 35 & 8 & - & 57 & 76 & 282 \\
\hline & $\mathbf{R}$ & 23 & 26 & 30 & 4 & 4 & 50 & 10 & 11 & 22 & 41 & 221 \\
\hline & & & & & & & & & & & & \\
\hline \multirow[t]{2}{*}{6} & $\mathbf{E}$ & 238 & 190 & 78 & 38 & 130 & 43 & 241 & 10 & 82 & 165 & 1215 \\
\hline & $\mathbf{R}$ & 244 & \begin{tabular}{|l|}
62 \\
\end{tabular} & 58 & 18 & 85 & 42 & 236 & 4 & 48 & 133 & 930 \\
\hline & & & & & & & & & & & & \\
\hline \multirow[t]{2}{*}{7} & E & 36 & 25 & 24 & 8 & 14 & 10 & 53 & 1 & 21 & 51 & 243 \\
\hline & $\underline{R}$ & 40 & 28 & 24 & 8 & 26 & 24 & 31 & 2 & 29 & 36 & 248 \\
\hline & & & & & & & & & & & & \\
\hline \multirow[t]{2}{*}{8} & $E$ & 4 & 6 & 1 & - &. & - & $=$ & - & 1 & 13 & 25 \\
\hline & $\mathbf{R}$ & 2 & 1 & 1 & - & . & 1 &. & 1 & . & 7 & 13 \\
\hline & & & & & & & & & & & & \\
\hline \multirow[t]{2}{*}{9} & $\mathbf{E}$ & - & $=$ & - & $=$ &. &. &. & - & 1 & 2 & 3 \\
\hline & $\mathbf{R}$ &. & - & $=$ & - &. & . & - &. &. & - & - \\
\hline & & & & & & & & & & & & \\
\hline \multirow[t]{2}{*}{10} & $\mathbf{E}$ &. & - & $\therefore$ & - & 1 & . & - & - &. & 4 & 5 \\
\hline & $\mathbf{R}$ & 5 &. & $\therefore$ &. & 2 &. &. &. &. & 5 & 12 \\
\hline & & & & & & & & & & & & \\
\hline \multirow[t]{2}{*}{11} & $\mathbf{E}$ & 21 & 9 & 10 & 7 & 21 & 15 & 18 & 3 & 10 & 16 & 130 \\
\hline & $\mathbf{R}$ & 25 & 8 & 17 & - & 2 & 5 & 15 & - & 3 & 8 & 83 \\
\hline & & & & & & & & & & & & \\
\hline \multirow[t]{2}{*}{12} & $\mathbf{E}$ & 3 & 7 & 3 & - & $\therefore$ & 1 & - &. & 2 & 8 & 24 \\
\hline & $\mathbf{R}$ & 4 & 1 & 4 & 1 & 1 & - & 5 &. & 1 & 12 & 29 \\
\hline TOTAL & & 733 & 436 & 333 & 98 & 346 & 247 & 698 & 38 & 306 & 659 & 3894 \\
\hline \multicolumn{13}{|c|}{$\begin{array}{l}\text { * } E \text { Q Quantidade de unidades de interaçáo emitidas pelo paciente estudado a outros } \\
\text { pacientes. } \\
R \text { = Quantidade de unidades de interação recebidas pelo paciente estudado, de outros } \\
\text { pacientes. }\end{array}$} \\
\hline
\end{tabular}

É possível que o fato de sua comunicação com o paciente efetuar-se eminentemente na área de tarefa, signifique para o grupo da enfermagem que esta deva ser a área prioritária de atenção a ser devotada ao paciente. Pode ser ainda que o paciente fique satisfeito com este nível de comunicação que recebe. Por outro lado, é possível também que ele fíque frustrado, que 
queira mais do que apenas ser solicitado para colaborar em procedimentos aos quais vai ser submetido com vistas ao seu tratamento, e na mesma proporção deseje receber atenção em relação aos seus medos, preocupaçōes e ansiedades.

O diálogo transcrito a seguir, ilustra a necessidade de informaçōes manifestadas por um paciente (A) internada no quarto da paciente número dois $(\mathrm{B})$ :

B - Será que essa operaçãozinha vai atrapalhar eu apanhar café?

A - Acho que tem que tirar os pontos primeiro.

A senhora vai tirar lá na cidade onde mora mesmo?

B - Vou. Não vou voltar aqui só para tirar os pontos não!

A - A senhora tem que apanhar café?

B - Tenho, lá na fazenda onde mora minha filha.

Ontem na hora de tirar aquele soro doeu tanto...

A - Por que não colocou no braço?

B - Porque eu tenho as veias do braço fracas.

Estouram muito. A vida toda foi assim.

A - A senhora que foi bom! Foi cedinho para a cirurgia. Assim é melhor!

B - Ontem quando eu desci para a sala tinha um monte de cama com ente que ia operar lá embaixo...

A - Tudo ia operar?

B - Tudo. Aí me deram uma injeção. Eu achei que já era a anestesia, mas disseram que não, que era só um remedinho. Depois me colocaram um soro e me passaram para outro lugar e aí eu já tava meio com sono. Ágora, na sala de operação eu já não vi mais nada. Já devo ter chegado dormindo.

A - Eles falam que a sala de operação ninguém vê, né?

B - É, ninguém vê mesmo. Eu acho que a anestesia eles colocam no soro.

A - É, eu acho que é no soro mesmo. Já ouvi muita gente que operou falar isso! 
Neste momento entrou na enfermaria a atendente do centro cirúrgico com a maca para levar OPA para a cirurgia. $\mathrm{E}$ a paciente número dois comentou com uma terceira:

B "Coitada! A dona Nair vai morrer de medo. Ela estava com tanto medo a noite inteira..."

O grupo da enfermagem poderia suprir esta necessidade de informação e apoio no pré-operatório se estivesse mais presente, mais próximo do paciente. A proximidade aliada à sensibilidade aos problemas e inquietações dos pacientes favoreceriam a comunicação.

Segundo AASTERUD ${ }^{1}$, a expansão do conhecimento sobre o comportamento das pessoas sob stress psicológico e físiológico tem trazido suportes que podem ajudar a enfermeira no julgamento sobre o tipo e a intensidade de informação que ela deve fornecer aos pacientes. Ressalta a autora, que a incorporação deste conhecimento e a identificação de fatores que podem bloquear a comunicaçāo enfermeiro-paciente, pode trazer não apenas benefícios para o paciente, mas também estímulo para a enfermeira, no sentido de prestar uma assistência baseada em resultados de investigação.

$\mathrm{Na}$ ausência deste apoio, desta presença constante da enfermeira, os pacientes recorrem uns aos outros para apresentar queixas, preocupações e para pedir ajuda, sugestões, informaçōes.

\begin{tabular}{|c|c|c|c|c|}
\hline \multicolumn{5}{|c|}{$\begin{array}{l}\text { TABELA } 2 \text { - Distribuição das Unidades de Interaçáo Trocadas entre Pacientes Estudados e } \\
\text { outros Pacientes, segundo Pacientes e Áreas de Categorias de Bales. } \\
\text { As Quantidades Superior e entre Parênteses correspondem, respectivamente, à Freqüência } \\
\text { Observada è Porcentagem. }\end{array}$} \\
\hline \multirow[t]{2}{*}{ PACIENTES } & \multicolumn{3}{|c|}{ ÁREAS } & \multirow[t]{2}{*}{ TOTAL } \\
\hline & POSITIVA & NEUTTRA & NEGATIVA & \\
\hline 1 & 55 & 620 & 58 & 733 \\
\hline & $(7,5)$ & $(84,6)$ & $(7,9)$ & $(100)$ \\
\hline 2 & $\begin{array}{c}20 \\
(4,6)\end{array}$ & $\begin{array}{c}391 \\
(89,7)\end{array}$ & $\begin{array}{c}25 \\
(5,7)\end{array}$ & $\begin{array}{c}436 \\
(100)\end{array}$ \\
\hline 3 & $\begin{array}{c}44 \\
(13,2)\end{array}$ & $\begin{array}{c}255 \\
(76,6)\end{array}$ & $\begin{array}{c}34 \\
(10,2)\end{array}$ & $\begin{array}{c}333 \\
(100)\end{array}$ \\
\hline 4 & 2 & 88 & 8 & 98 \\
\hline & $(2,0)$ & $(89,8)$ & $(8,2)$ & $(100)$ \\
\hline 5 & $\begin{array}{c}51 \\
(14,7)\end{array}$ & $\begin{array}{c}268 \\
(77,5)\end{array}$ & $\begin{array}{c}27 \\
(7,8)\end{array}$ & $\begin{array}{c}346 \\
(100)\end{array}$ \\
\hline 6 & $\begin{array}{c}15 \\
(6,1)\end{array}$ & $\begin{array}{c}211 \\
(85,4)\end{array}$ & $\begin{array}{c}21 \\
(8.5)\end{array}$ & $\begin{array}{c}247 \\
(100)\end{array}$ \\
\hline 7 & 76 & 584 & 38 & 698 \\
\hline 8 & $\begin{array}{c}(10,9) \\
6\end{array}$ & $\begin{array}{c}(83,7) \\
29\end{array}$ & $\begin{array}{c}(5,4) \\
3\end{array}$ & $\begin{array}{c}(100) \\
38\end{array}$ \\
\hline & $(15,8)$ & $(76,3)$ & $(7,9)$ & $(100)$ \\
\hline 9 & $\begin{array}{c}27 \\
(8,8)\end{array}$ & $\begin{array}{c}263 \\
(85,9)\end{array}$ & $\begin{array}{c}16 \\
(5.2)\end{array}$ & $\begin{array}{c}306 \\
(100)\end{array}$ \\
\hline 10 & 46 & 527 & 86 & 692 \\
\hline TOTAL & $\begin{array}{c}342 \\
(8.8)\end{array}$ & $\begin{array}{c}3236 \\
(83.1)\end{array}$ & $\begin{array}{c}316 \\
(8.1)\end{array}$ & $\begin{array}{l}3894 \\
(100)\end{array}$ \\
\hline
\end{tabular}


É o que se depreende dos resultados desta pesquisa, conforme demonstram as Tabelas 1 e 2 . Nesta última tabela verifica-se que o padrão de interação dos pacientes entre si, segue o mesmo já identificado em estudos anteriores realizados por MENDES ${ }^{10,11,12,13}$, para os integrantes do grupo da enfermagem: enfermeiros, auxiliares e atendentes de enfermagem, ou seja, uma comunicação essencialmente vinculada à área neutra $(83,1 \%)$, com pequeno percentual de unidades de interação classificado na área positiva $(8,8 \%)$ e negativa $(8,1 \%)$.

Este resultado é compatível com as demandas instrumentais das situações clínicas, mas não se compatibilizam com as demandas interpessoais destas mesmas situações. Quando se correlaciona tais resultados com os conceitos adotados teoricamente acerca da essencialidade da comunicação no processo de enfermagem, verifica-se existir aí um paradoxo. $O$ intenso compromisso para com as tarefas, caracteriza uma modalidade de assistência de enfermagem que não abre espaço para um conteúdo de comunicação na área sócio-emocional; e o conteúdo da comunicação retrata a primazia da tarefa e reflete-se também na comunicação paciente-paciente.

Conforme demonstrado por $\mathrm{MENDES}^{9}$, cria-se uma barreira entre o grupo de pacientes e o grupo de enfermagem " $E$ as barreiras se elevam justamente por causa da atitude tomada pelo grupo da enfermagem: 1) executando tarefas em grupo; 2) não interagindo verbalmente com os pacientes na maioria das vezes em que trabalham em grupo numa enfermaria, em que cada um faz sua parte da tarefa executada no mesmo paciente; 3 ) interagindo entre si sobre assuntos e interesses particulares que nada dizem respeito ao paciente, que permanece exposto ao humor do funcionário.

Desta forma, percebendo a união dos elementos do grupo da enfermagem, o grupo de pacientes também se une, fechando entre si muito sentimento, muito emoçāo e muita angústia, muita agressividade e algumas alegrias que sāo na maioria das vezes, desconhecidas pelo pessoal de enfermagem".

Neste contexto, a afirmação de MULDARY ${ }^{16}$ faz sentido ao lembrar que todo comportamento pode ter um valor de comunicação que pode ou não ser reconhecido; este valor dependerá de ser esta comunicação percebida ou não, e de ser a ela atribuído um significado. Portanto, é preciso que se tenha sempre em perspectiva, que o comportamento humano não resulta em comunicação se não for percebido e se não tiver significado.

\section{CONCLUSÃO}

O padrão de comunicação entre pacientes num hospital governamental brasileiro, configura-se condizente com a modalidade funcional de assistência, que prioriza a tarefa em detrimento das relações inter- 
pessoais. Assim, a comunicação dos pacientes entre si revelou o seguinte padrão: das 3894 unidades de interação trocadas entre eles, $3236(83,1 \%)$ foram classificadas na área neutra; $342(8,8 \%)$ na área positiva e 316 $(8,1 \%)$ na área negativa.

É preciso que se repense o valor da comunicação no processo de enfermagem e que os enfermeiros percebam e interpretem a comunicação que ocorre entre seus pacientes, fazendo intervençōes no sentido de criar condições para que manifestem suas necessidades, que então poderão ser melhor atendidas.

MENDES, I.A.C. et al. Pattern of communication between patients in a Brazilian government hospital. Rev. Esc. Enf. USP., v. 27, n.3, p. 403-12, dec. 1993.

In a situation of verbal interaction in hospital nursing, the authors explore the context of the patient with the objective of verifying the pattern of communicaton between patients. The data were obtained by direct observation of the verbal behavior of a sample of 10 patients with their hospital roommates. The data were analyzed by judges and classified in light of the Bales instrument. The results point to the following pattern of communication: $83,1 \%$ neutral interaction, $8,8 \%$ positive social-emotional interaction, and $8,1 \%$ negative social-emotional interaction. The authors discuss these results, stimulating nurses to reassess the value of communication in the nursing process.

UNITERMOS: Nurse. Patient relations.

\section{REFERÊNCIAS BIBLIOGRÁFICAS}

1. AASTERUD, M. Explanation to the patient. Nurs.Forum, v.11, n.4, p. 37-45, 1963.

2. BALES, R.F. Interaction process analysis : a method for the study of small groups. Cambridge, Addison-Wesley-Press, 1950.

3. BEANLANDS, H.E.; MACKAY, R.C. Nurse, do you hear me? Can.Nurse, v.77, n.7, p.41-3, 1981.

4. CLARK, J.M. Communication in nursing. Nurs.Times, v.77, n.1, p. 12.8, 1981.

5. CONANT, L.H. Give-and-take in home visits. Am.J.Nurs., v.65, n.7, p. 117-20, 1965

6. GIR, E. et al. Análise da interação verbal enfermeiro-paciente aidético através do sistema SAVI. IN: SIMPÓSIO BRASILEIRO DE COMUNICAÇĀO EM ENFERMAGEM,2, Ribeirão Preto, 1990. Anais. Ribeirão Preto. Escola de Enfermagem de Ribeirâo Preto,Universidade de Sáo Paulo, 1990.

7. LANGLOIS, M.S. La relation du rôle joué par le personnel infirmier sur la prise de rôle de la personne âgée en établissement de soins prolongés. Nurs.Pap., v.17, n.1, p. 48-65, 1985.

8. McCORKLE,R. Effects of touch on seriously ill patients. In: Fox. D.J.;_Readings on the research process in nursing, New York, Appleton-Century Crofts, p. 114-25, 1981.

9. MENDES, I.A.C. Interação verbal em situaçōes de enfermagem hospitalar: enfoque humanístico, Ribeiräo Preto, 1986. 175p. Tese (Doutorado)-Escola de Enfermagem de Ribeirāo Preto, Universidade de Sāo Paulo.

10. MENDES, I.A.C. et al. O padrão de comunicação do enfermeiro com o paciente. Rev.Paul.Enf., v.8, n.1, p. 13-6, 1988. 
11. MENDES, I.A.C. et al. Matriz de interaçāo: auxiliar de enfermagem e paciente. Rev.Gaúcha Enf., v.9, n.1, p. 43-6, 1988.

12. MENDES, I.A.C. et al. Perfil de interação: atendente de enfermagem e paciente. IN: SIMPÓSIO BRASILEIRO DE COMUNICAÇÁO EM ENFERMAGEM, 1, . Ribeirão Preto, 1988. Anais Ribeirão Preto, 1988. Escola de Enfermagem de Ribeirão Preto, Universidade de São Paulo, 1988. p.327-37.

13. MENDES, I.A.C. et al. A utilização do sistema de Bales no estudo da interação enfermeiro-paciente. Rev.Esc.Enf.USP., v.23, n.3, p. 295-303, 1989.

14. MENDES, I.A.C. et al. Comunicaçāo com o paciente: o teórico e o concreto na enfermagem de âmbito hospitalar. Rev.Bras.Enf., v.44, n.2/3, p. 98-102, 1991.

15. MORIMOTO, F. Favoritism in personnel patient interaction. Nurs.Res., v.3, n.3, p.109-12, 1955.

16. MULDARY, T.W. Interpersonal relations for health professional: a social skills approach. New York. Macmillan Publishing, 1983.

17. NIGHTINGALE, F. Notes on nursing: what it is and what it is not. London. Gerald Duckworth, 1970.

18. SAWADA, N.O. et al. Interação enfermeiro-paciente: dimensão não-verbal durante a pré-anestesia. IN: SIMPÓSIO BRASILEIRO DE COMUNICAÇÃO EM ENFERMAGEM, 2, Ribeirão Preto, 1990. Anais. Ribeirão Preto, Escola de Enfermagem de Ribeirāo Preto, Universidade de São Paulo, 1990. p.391-410.

19. SILVA, M.J.P. et al. Como os enfermeiros percebem a comunicação na interação enfermeiro-paciente. IN: SIMPÓSIO BRASILEIRO DE COMUNICAÇÄO EM ENFERMAGEM, 2, Ribeirão Preto, 1990. Anais. Ribeirão Preto,Escola de Enfermagem de Ribeirão Preto, Universidade de Sāo Paulo, 1990. p.374-90.

20. SPRING, F.E.; TURK, H. A terapeutic behavior scale. Nurs.Res., v.11, p.4, p.214-8, 1962.

21. STETLER, G.R. Relationship of perceived empathy to nurses communication. Nurs.Res., v.26, n.6, p. 432-8, 1977. 